\title{
THREE-DIMENSIONAL EVALUATION OF THE TEMPOROMANDIBULAR JOINT AFTER USING FACEMASK APPLIANCE; A CLINICAL PROSPECTIVE STUDY
}

\author{
Hanem Y. El Feky* and Adel Rashid *
}

\begin{abstract}
Aim of the study: This study was conducted to evaluate the three dimensional changes in the TMJ after the maxillary protraction using face mask therapy.

Material and Methods: Eighteen growing patients (ages ranged from 8-11 years) were included in the current study. All patients manifested features of skeletal class III due to maxillary deficiency. A Petit-type facemask was used for all patients and was attached intraorally to a fixed labiolingual appliance through extraoral elastics .A cone-beam CT scan had been applied for each patient before the start of the treatment and after 10 months. The three-dimensional (3D) temporomandibular joint measurements included: 1) anterior fossa line inclination, 2) posterior fossa line inclination, 3) anterior joint space, 4)posterior joint space,5) medial joint space, and 6) superior joint space.

Results : Regarding Anterior Joint space : measurements after using a facemask appliance were significantly higher than before (as $\mathrm{P}<0.05$ ), while regarding Posterior and superior Joint spaces: measurements after using a facemask appliance were significantly lower than before $($ as $\mathrm{P}<0.05)$

Conclusion: Facemask therapy for maxillary protraction in management of skeletal class III produced: 1- Decrease in both posterior and superior joint spaces, while increase in the anterior joint spaces. 2- Bone remodeling in the glenoid fossa causes upward and backward displacement of the condyle.
\end{abstract}

KEYWORDS: Facemask , Tempromandibular joint , Maxillary protraction

\section{INTRODUCTION}

Class III malocclusion is one of the most difficult problems to treat in the mixed dentition ${ }^{(1)}$. It has a multifactorial etiology involving genetic, habitual, ethnic, and environmental components ${ }^{(2-4)}$.
Different ethnic groups exhibit different prevalence rates of Class III. The prevalence rate was reported to be around $1-3 \%$ in the Caucasians and around 13-14\% among the Chinese and Japanese. In the Asian population the majority of patients exhibit midface deficiency ${ }^{(5)}$.

\footnotetext{
* Lecturer of Orthodontics, Faculty of Dentistry, Fayoum University, Egypt.
} 
Patients with Class III malocclusion can exhibit maxillary retrusion , mandibular protrusion, or both, together with abnormal dental relationship problems and facial esthetic disorders. Further, on an average, $60 \%$ of Class III malocclusions are characterized by maxillary deficiency ${ }^{(6)}$.

Awareness of maxillary deficiency as the main contributing part of the Class III structural etiology, has led to using various devices with an orthopedic effect: face mask (FM)/orthopedic mask , protraction headgear/reverse headgear, chin cup, reverse twinblock, and skeletal anchorage systems ${ }^{(7-9)}$.

All these orthopedic appliances are effective in treating the skeletal maxillary deficiency in growing patients. However, many studies show that the effects depend largely on the timing of the treatment: the earlier the treatment is applied, the more the orthopedic effects on the maxillary growth and the less the unwanted dental changes (orthodontic effect).

Most of the studies of protraction facemask (PFM) treatment mainly focused on its efficacy on Class III malocclusion, including the skeletal and dental changes ${ }^{(10-14)}$, but few ones were available concerning the effect of PFM on TMJs and The interaction between the mandibular condyle and the glenoid fossa subsequent to its treatment ${ }^{(15-18)}$.

In the orthodontic practice, cephalometric and panoramic radiographs are the most common approaches in the radiographic evaluation of the TMJ because of availability, ease of use, low radiation requirement, and low cost ${ }^{(19)}$. However, validity of $2 \mathrm{D}$ imaging is doubtful depending on the changes created by patients' head position and beam projection angle. Furthermore, anatomic superimposition and magnification differences of the left and right sides that cause double border of the mandible on the radiograph are the other disadvantages ${ }^{(20)}$.
Recent improvements in technology have led to the $3 \mathrm{D}$ cone beam computed tomography (CBCT) that has been developed specifically for the maxillofacial region. CBCT could provide sub-millimeter spatial resolution images with markedly shorter scanning times and has been reported to require somewhat lower radiation dosages than computed tomography (CT) imaging methods. CBCT allows personal computer-based two dimensional (2D) multi-planar reformatted (MPR) and secondary reconstruction of the data as well as volumetric three dimensional (3D) images that allows 1:1 image reality ${ }^{(21)}$.For this reason, this study was conducted to evaluate the three dimenetional changes in the TMJ before and after the maxillary protraction using face mask therapy.

\section{MATERIALS AND METHODS}

\section{A-Subjects}

Eighteen growing patients were included in the current study selected from the economic outpatients orthodontic clinics, Faculty of Dentistry, Fayoum University, Fayoum, Egypt .

\section{Inclusion criteria}

Growing patients with ages ranging from 8- 11 years were selected with the features of the prepubertal cervical vertebrae maturational stages 1 \& 2 (initiation \& acceleration), and were assessed according to the method of Hassel \& Farman ${ }^{(22)}$ on the pre-treatment cone beam computed tomogram (CBCT).

All patients manifested features of skeletal class III due to maxillary deficiency as verified both clinically \& radiographically, which included concave or flat facial profile, ANB angle $\leq 0^{\circ}$, SNA angle $\leq 80^{\circ}$, Anterior cross bite or an edge to edge incisor relationship, Angle class III molar relationship, and Presence of fully erupted maxillary first permanent molars. 


\section{B-Methods}

Patients who met the eligibility criteria during recruitment were invited to participate in the current study which was carried out at Faculty of Dentistry, Fayoum University. Approval for the research proposal was obtained from Ethics Committee of the Fayoum University \{Number (EC2113) in February2021 $\}$ All the clinical procedures of the study and its aim were explained in details for all the patients individually and written consent terms were obtained from the patients and legal guardians.

\section{1-Clinical procedure:}

A Petit-type facemask (Ormco, USA) was used in this study. Intraorally the facemask was attached to a fixed labiolingual appliance through extraoral elastics. The fixed intraoral labiolingual appliance consisted of palatal arch (1mm stainless steel) soldered to two bands adapted on the two maxillary permanent molars. One hook was soldered to each band labially and directed distally. Sizes of the elastics were selected individually for each patient in order to provide $400 \mathrm{~g}$ of traction force per side. The traction direction of the elastic pull was set as $30^{\circ}$ to the occlusal plane (Figures 1 and 2 ).

The patients were informed by the instructions as to wear the appliance 12-16 hours per day (afterschool hours and during sleep). The patients were also instructed to change the elastics daily and whenever they experienced a loss of the elastic. The treatment continued for a period of 10 months.

\section{2-CBCT Imaging :}

A cone-beam CT scan (i-CAT Next generation: imaging sciences international, Hatfield, PA) had been applied for each patient before the start of the treatment and after 10 months.

It was prudent to use a CBCT scanner that respects the ALARA principle, and handle the data with a software capable of maximizing the output.

\footnotetext{
* Anatomage, San Jose, CA, USA.
}

The CBCT scanner used in the current study was a flat panel measuring $17 \times 23 \mathrm{~cm}$, which produces less radiation dose at $120 \mathrm{kV}, 18.54 \mathrm{mAs}$, and 8.9 seconds than other flat-panel detectors based systems. the effective dose is $74 \boldsymbol{\mu S v}$.

The CBCT scans were done according to the following protocol: Extended field of view (FOV) $17 \mathrm{x}$ $23 \mathrm{~cm}$, a voxel size of $0.3 \mathrm{~mm}$. The patient's head was oriented with the Frankfurt Horizontal plane parallel to the floor and perpendicular mid-sagittal plane to the floor. CBCT scans were obtained in a Digital Imaging

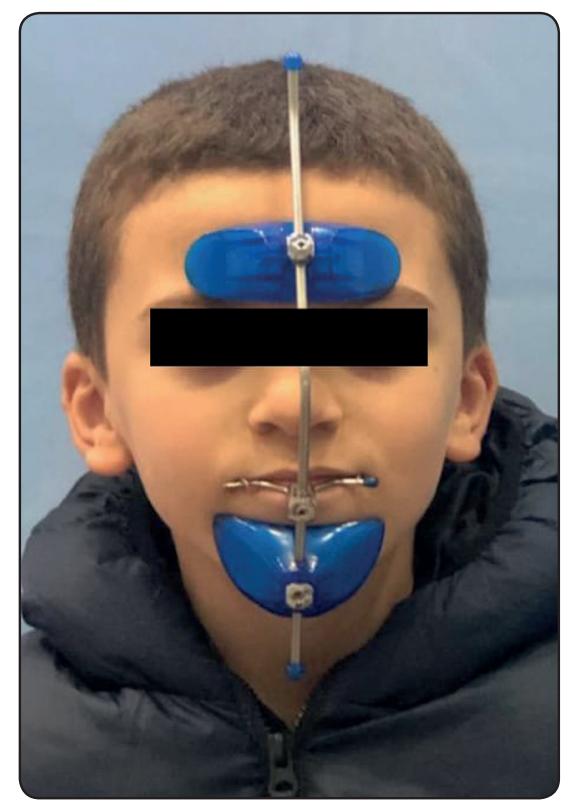

Fig. (1): A Petit-type facemask in plac

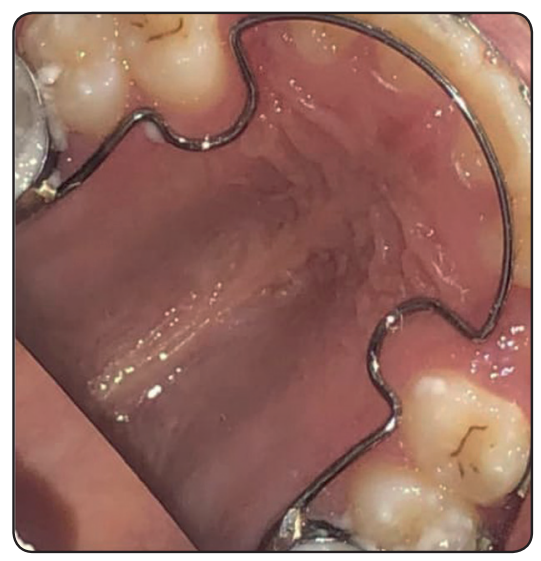

Fig. (2): The Intraoral component of the facemask ; the fixed labiolingual applianc 


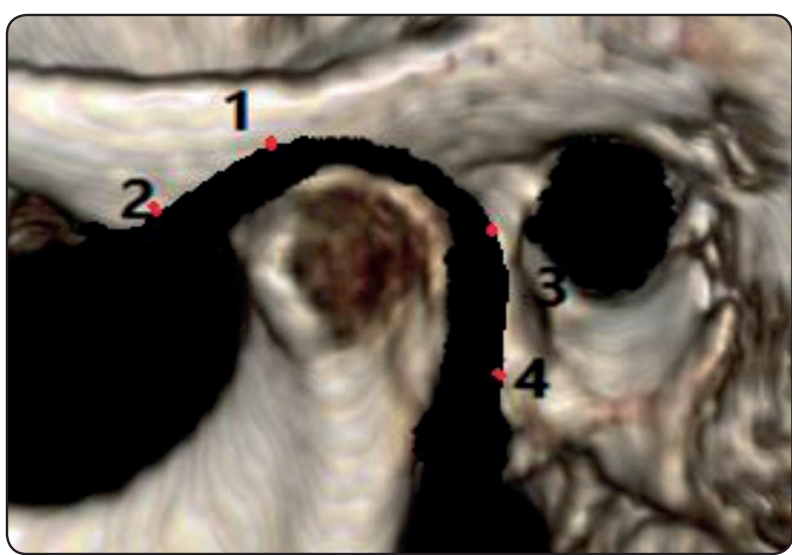

Fig. (3): Volumetric sagittal view of the TMJ showing the mandibular fossa points; $1-\mathrm{FL}_{\mathrm{a}-\mathrm{s},}$, $\mathrm{FL}_{\mathrm{a}-\mathrm{i}}, 3-\mathrm{FL}_{\mathrm{p}-\mathrm{s}}$ and 4- $\mathrm{FL}_{\mathrm{p}-\mathrm{i}}$. and Communications in Medicine (DICOM) format. Cone beam computed tomography images were converted to Dicom format and processed into volumetric images using Anatomage image processing software version $5.01^{\mathrm{g}}$. Multiplanar sagittal, coronal and axial projections were generated*.

\section{3-Temporomandibular joint analysis:}

The TMJ analysis was done according to Alhammadi et al (23) and similar to Alfeky (24) Craniofacial and TMJ selected points were identified three dimensionally, Lines and planes were constructed, and selected linear and angular measurements were computed and recorded. The three-dimensional (3D) temporomandibular joint

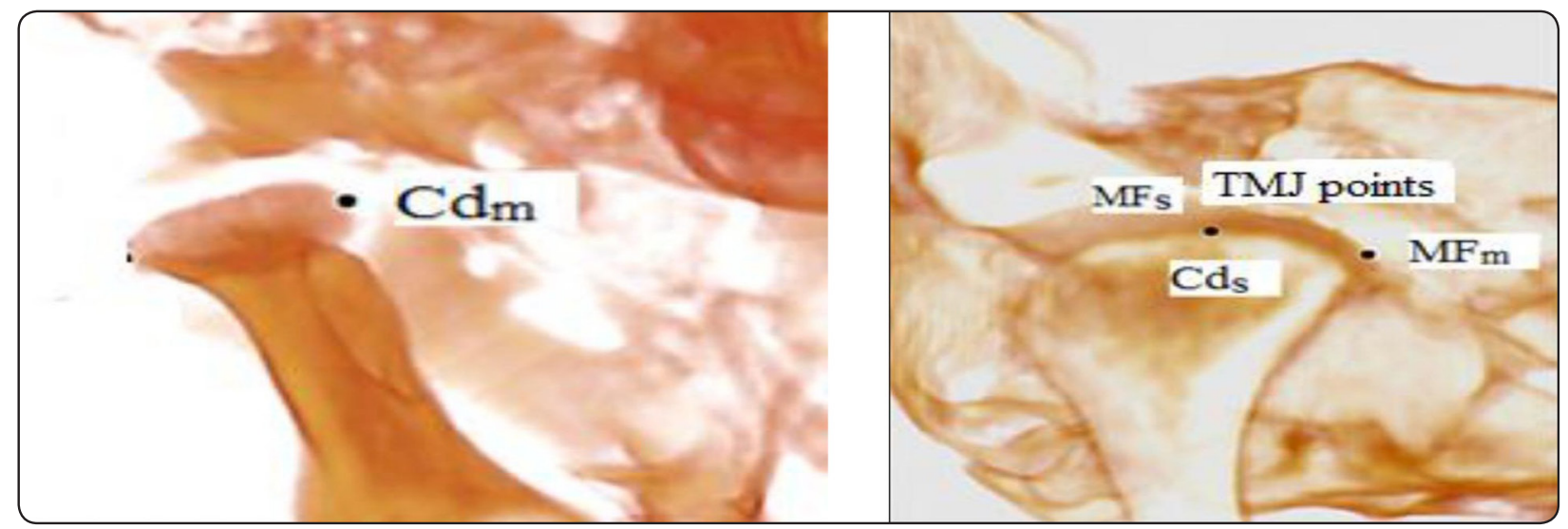

Figure (4): Volumetric sagittal view of the TMJ showing the mandibular fossa \& condylar points; $\mathrm{MF}_{\mathrm{s}}, \mathrm{MF}_{\mathrm{m}}, \mathrm{Cd}_{\mathrm{s}}$, and $\mathrm{Cdm}$.

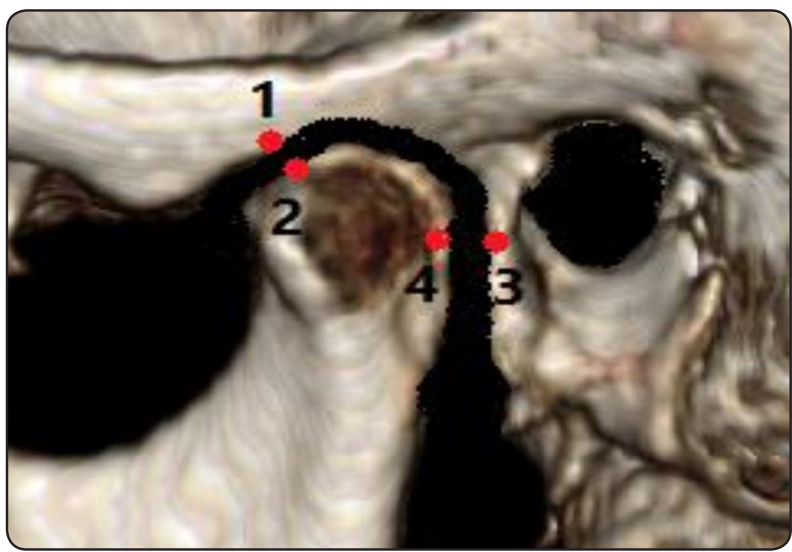

Fig. (5): Volumetric sagittal view of the TMJ showing the anterior \& posterior joint spaces landmarks used; 1$\mathrm{JSa}_{\mathrm{f}}, 2-\mathrm{JSa}_{\mathrm{c}}$, 3- JSp-f and 4- JS $\mathrm{p}-\mathrm{c}^{\text {. }}$

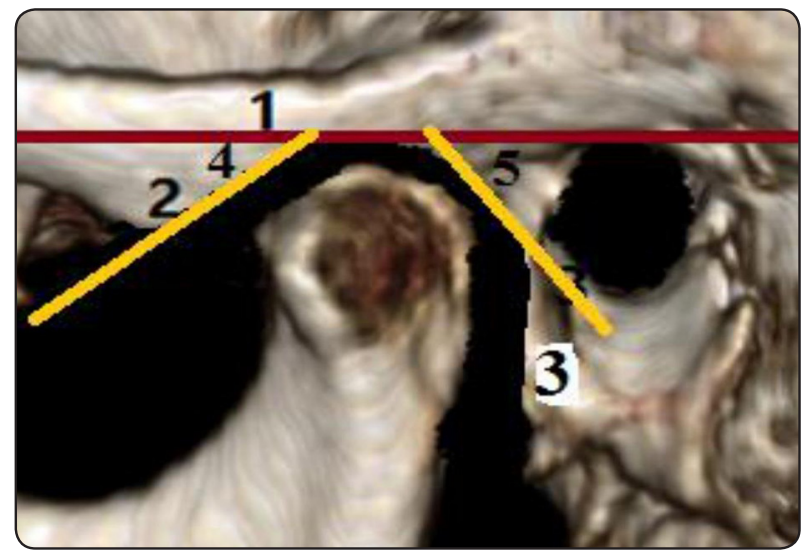

Fig (6): Sagittal view of the condyle and mandibular fossa showing; 1) FHP, 2) AFL, 3) PFL, 4) AFLIFHP angle, and 5)PFL/FHP angle. 


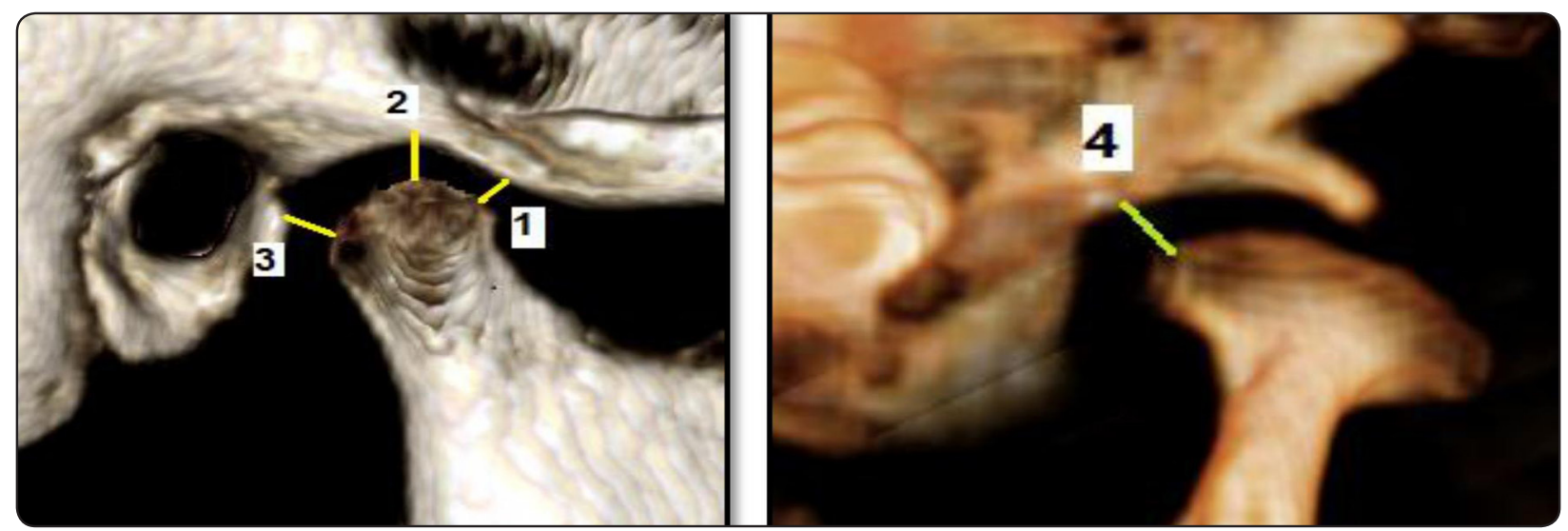

Fig. (7): Sagittal view of the condyle showing the 1 ) anterior, 2) superior, 3) posterior joint, and 4) medial spaces measurements

measurements included: 1) anterior fossa line inclination, 2) posterior fossa line inclination, 3) anterior joint space,4) posterior joint space,5) medial joint space, and 6) superior joint space. Descriptions of these points, lines and measurements are listed in table (I, II).

\section{Statistical analysis:}

Statistical analysis was performed with SPSS 16® (Statistical Package for Scientific Studies), Graph pad prism \& windows excel. Exploration of the given data was performed using Shapiro-Wilk test and Kolmogorov-Smirnov test for normality which revealed that the significant level (P-value) was insignificant as P-value $>0.05$ which indicated that alternative hypothesis was rejected, and the concluded data originated from normal distribution (parametric data) resembling normal Bell curve.

\section{RESULTS}

Inter-observer reliability coefficient (Kappa test) was used to evaluate the agreement between the same assessor observations. It revealed almost perfect agreement (IOC> 0.65) regarding all the measurements.

Clinical improvement in the form of increased convexity in the soft profile before and after maxillary protraction using facemask therapy was shown in figure (8).

\section{A-The assessment of the TMJ before and after facemask appliance therapy:}

The three-dimensional (3D) temporomandibular joint measurements in both right and left sides including: anterior fossa line inclination, posterior fossa line inclination, anterior joint space, posterior joint space, medial joint space and superior joint space were performed in presented in table (III) and figures $(9,10)$.

\section{B-Comparison between before and after was per- formed using Paired t-test which revealed that:}

\section{In the right side,}

1. Regarding Anterior Joint space : AJS measurement was significantly increased after using a facemask appliance than before as $\mathrm{P}<0.05$.

2. Regarding angle between Posterior fossa line / and Frankfort horizontal plane, Posterior Joint space and Superior Joint space: measurements after using a facemask appliance were significantly lower than before as $\mathrm{P}<0.05$.

3. On the other hand, there was insignificant difference between the angle between Anterior fossa line / Frankfort horizontal plane and Medial Joint space after the treatment as presented in table (IV) and figure (11). 


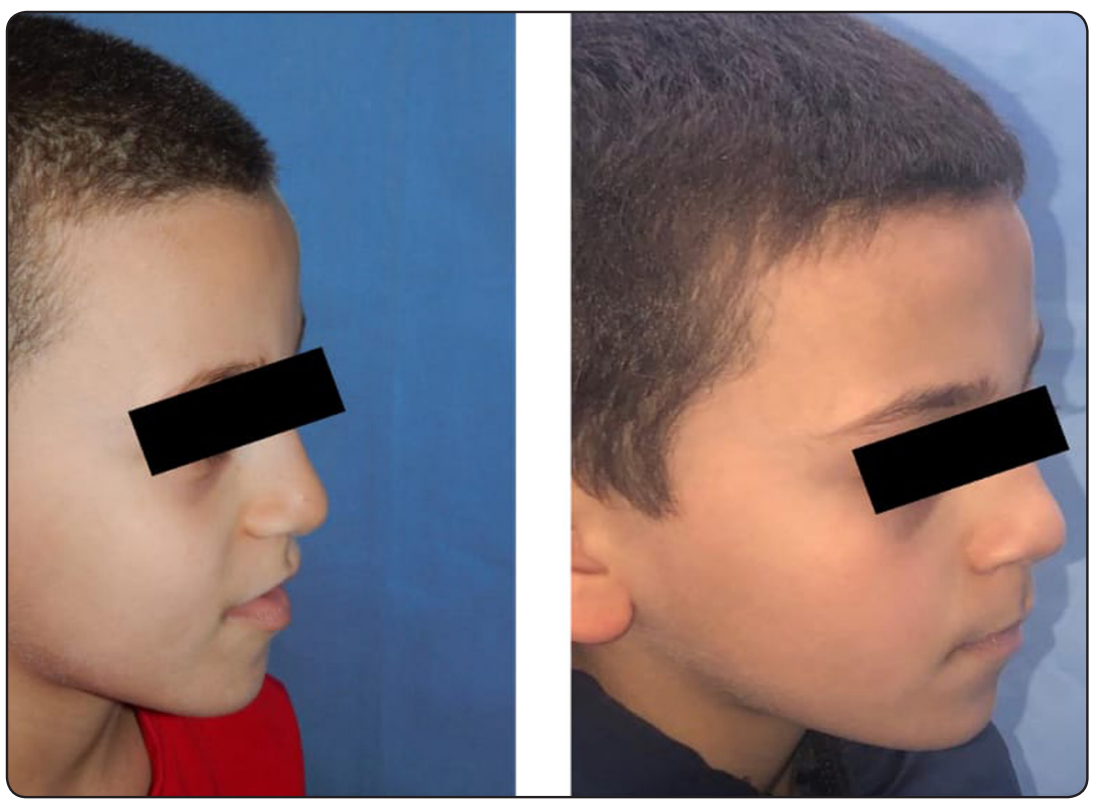

Fig. (8): Extroral photographs of a case of the facemask group showing increased convexity of the facial profile (A) before and (B) after the observation period.
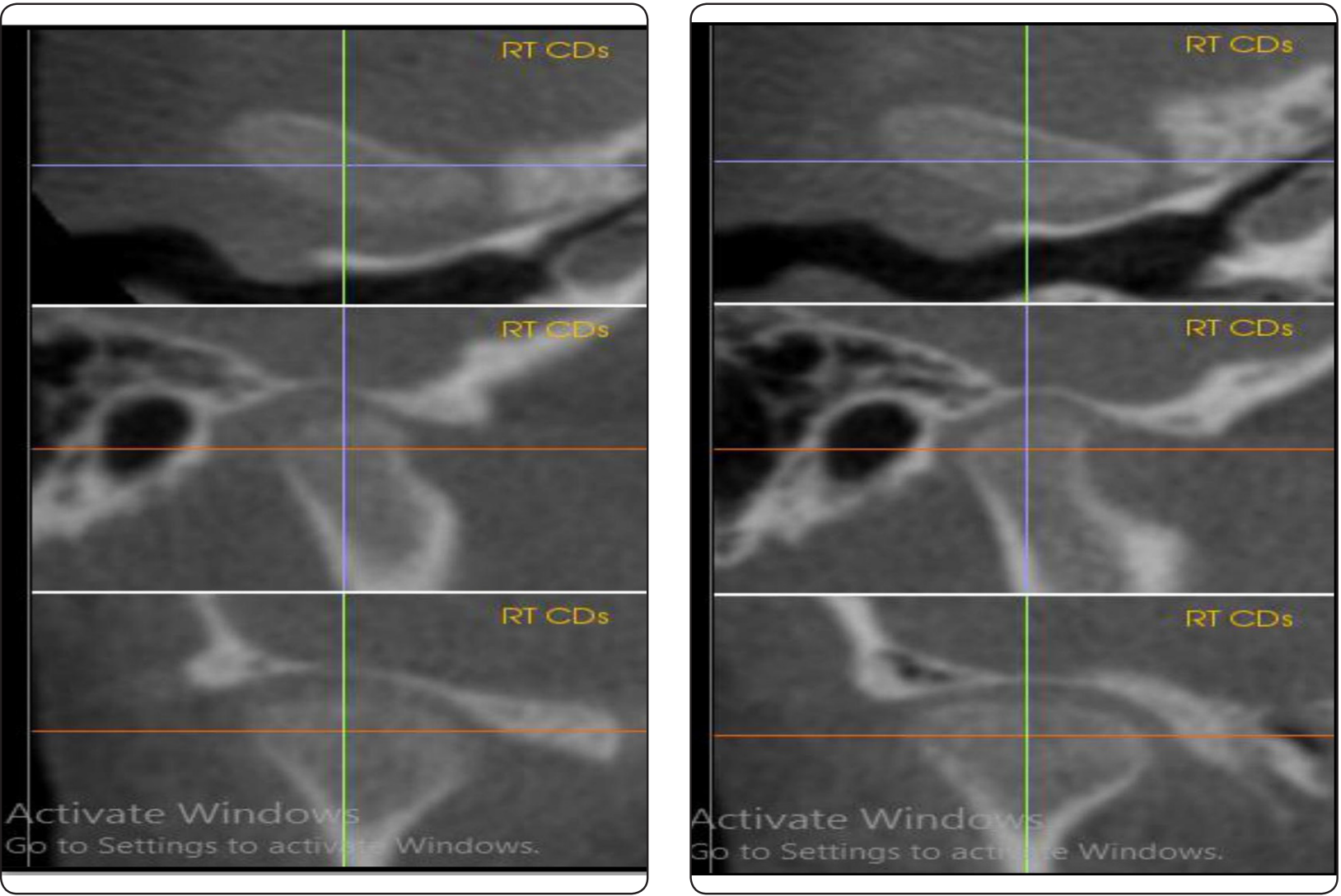

Fig. (9): Condylar position before maxillary protraction.

Fig. (10): Condylar position after maxillary protraction. 


\section{In the left side,}

1. Regarding Anterior Joint space: it was significantly increased after using a facemask appliance as $\mathrm{P}<0.05$.

2. Regarding Posterior Joint space and posterior fossaline/FHP: measurements were significantly lower after using a facemask appliance than before as $\mathrm{P}<0.05$.

3. On the other hand, there was insignificant difference between the angle between anterior fossa lines / Frankfort horizontal plane, superior Joint space and Medial Joint space after the treatment. As presented in table (IV) and figure (11).

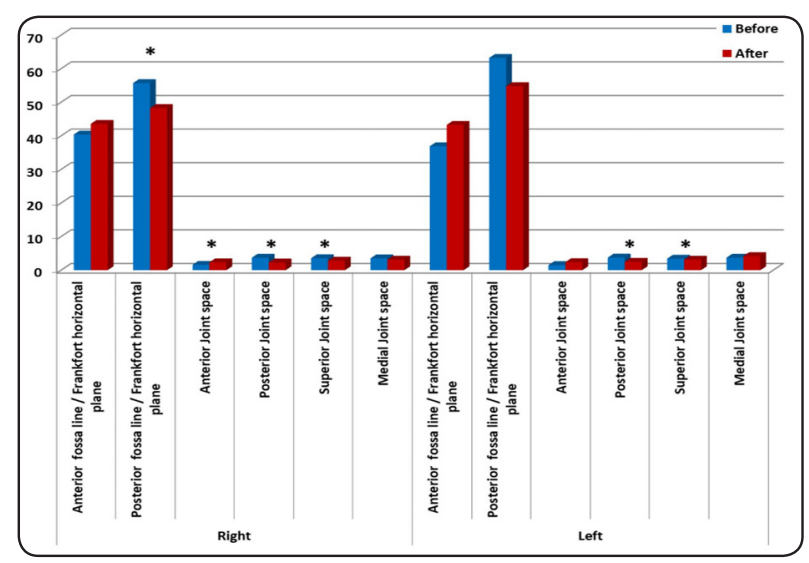

Fig. (11): Bar chart Comparison between mean right and left sides of anterior fossa line /Frankfort horizontal plane, posterior fossa line /Frankfort horizontal plane, anterior Joint space, posterior Joint space, superior Joint space and medial Joint space.

\section{DISCUSSION}

Application of the facemask appliances can produce good results in patients having skeletal Class III malocclusion due to maxillary deficiency, moving the maxilla and maxillary teeth forward and the mandible backward (25) .Several studies demonstrated that reverse headgear treatment stimulated forward and downward displacement of the maxilla by disrupting the circummaxillary sutures, so early treatment is commonly indicated in such cases ${ }^{(25-27)}$.

The reaction force s resulted from the forward movement of the maxillary complex tend to push the chin posteriorly. The final effects are clockwise rotation of the mandible and increase the vertical dimension. Anteroposterior positional changes in the temporomandibualr joint including the mandibular fossa and the positions of the condyle are expected ${ }^{(16)}$.

Prepubertal growing children with age range of 8-11 years were included in this study based on their cervical vertebrae maturational indicators where they have CVMI stages 1 or 2. A control group with the same malocclusion was not added to this study as it was thought to be unethical to deny the patients privilege of early intervention in such a particular condition.

The use of cone beam computed tomographic scans (CBCT) for cranio-maxillofacial regions has gained wide acceptance over the past decade due to its various applications in orthodontic diagnosis and treatment planning. ${ }^{21}$ The possibility of perceiving the third dimension has been greatly appreciated by orthodontists especially after realizing the shortcomings of the 2-D lateral cephalometric radiograph ${ }^{(20)}$.

CBCT has not been used frequently in the evaluation of condylar response to functional orthopedic therapy in patients with skeletal Class III malocclusion. Only Lee et al.in 2016 ${ }^{(17)}$ analyzed 3D TMJ changes in subjects with Class III malocclusion using facemask appliance, and De clerck et al., ${ }^{(16)}$ evaluated the condylar response to orthopedic therapy using bone anchored intermaxillary traction .

The TMJ analysis used in our study was adopted from the study of Alhammadi., ${ }^{(23)}$ and Alfeky ${ }^{(24)}$ who presented a full standardized custom made 3D analysis specifically for the TMJ. In order to properly and accurately assess the TMJ, the precise 
position, and other dimensions of the mandibular fossa and the mandibular condyles were measured. These measurements included inclination of the anterior and posterior wall of the mandibular fossa in relation to the Frankfort horizontal plane (AFL/ FHP, PFL/FHP), all joint spaces included superior, anterior, posterior, and medial joint space (SJS, AJS, PJS, MJS).

Regarding the mandibular fossa changes, it was noticed in the current study that the fossa was significantly posteriorly and superiorly displaced as revealed by the insignificant increase in their anterior walls inclination (AFL) and the significant decrease in their posterior walls inclination (PFL). The condyles were also significantly displaced posterior, and superior in the glenoid fossa as indicated by the statistically significant increase in the anterior joint space (AJS) and the significant decrease in the superior(SJS) and posterior joint spaces (PJS).

These findings were in accordance with those of De clerk et al., ${ }^{(16)}$ and Lee et al., ${ }^{(17)}$ who suggested that the compressive stresses of the face mask appliance on the mandible were located on the posterosuperior aspects of the mandibular fossa together with condylar head and tensile stresses on their anterior aspects. Therefore, new bone was deposited on the anterior wall of the fossa and the condyle and resorption occurred on the posterior walls of the fossa changing the fossa position and consequently the condyles were also displaced posterior and superior.

The present 3-D analysis of facemask therapy, confirmed that bone remodeling after facemask therapy for maxillary protraction leads to bone apposition to the anterior wall and bone absorption of the posterior walls of the glenoid fossa. The simultaneity of the displacement of the condyle and the above-noted bone remodeling results indicates that bone remodeling in the glenoid fossa causes upward and backward displacement of the condyle.

\section{CONCLUSIONS}

Facemask therapy for maxillary protraction in management of skeletal class III produced:

1. Bone apposition to the anterior wall and bone resorption of the posterior walls of the glenoid fossa.

2. Decrease in both posterior and superior joint spaces, while increase in the anterior joint spaces.

3. Bone remodeling in the glenoid fossa causes upward and backward displacement of the condyle.

\section{LIMITATION}

Although ethically we could not include prospective control group (growing patients with skeletal Class III with no treatment), and there was no historic control group available, thus the interpretation of results of this study should be performed with much caution, because we could not separate the changes presented whether it is purely from the intervention (facemask) or partly due to growth changes.

\section{REFERENCES}

1. Graber M., Van L, and Vig L. Treatment of patients in the mixed dentition, in Orthodontics: Current Principles and Techniques, Graber T and Vanarsdall R. 2nd Eds,2009; pp. 565-569, Mosby, St. Louis, Mo, USA .

2. Cozzani G. Extraoral traction and class III treatment . Am J Orthod Dentofacial Orthops,1981; vol. 80, no. 6, pp. $638-650$

3. De Toffol L, Pavoni C, Baccetti T, Franchi L and Cozza P . Orthopedic treatment outcomes in Class III malocclusion. A systematic review. Angle Orthod ,2008; 78, 561-573.

4. McNamara J. A. An orthopedic approach to the treatment of Class III malocclusion in young patients. Jo of Clin Orthod,1987; vol. 21, no. 9, pp. 598-608.

5. Bukhary M. T. Comparative cephalometric study of Class III malocclusion in Saudi and Japanese adult females. Jo of Ora Scie, 2005;vol. 47, no. 2, pp. 83-90. 
6. Ellis E and McNamara JA. Components of adult class III malocclusion. J Oral Maxillofac Surg. 1984;42:295-305.

7. Yavuz İ, Halıcıoğlu K and Ceylan İ. Face mask therapy effects in two skeletal maturation groups of female subjects with skeletal Class III malocclusions. Angle Orthod, 2009, 79(5): 842-848.

8. Kim JH, Viana MA, Graber TM, Omerza FF and BeGole EA. The effectiveness of protraction face mask therapy: a metaanalysis. Am J Orthod Dentofacial Orthop, 1990, 115(6):675- 685 .

9. Seehra J, Fleming PS, Mandall N and DiBiase AT. A comparison of two different techniques for early correction of Class III malocclusion. Angle Orthod, 2012, 82(1):96-101.

10. Ngan P. Early timely treatment of class III malocclusion. Semin in Ortho, vol. 11,2005; no. 3, pp. 140-145.

11. Delaire J. Manufacture of the orthopedic mask. Rev Stomatol Chir MaxilloFac, 1971;vol. 72, pp. 579-584.

12. Ngan P. Early treatment of Class III malocclusion: is it worth the burden. Am J Orthod Dentofacial Orthop, 2006; vol. 129, no. 4, supplement, pp. S82-S85.

13. Westwood PV, McNamara JA, Baccetti T, Franchi L and Sarver DM. Long-term effects of class III treatment with rapid maxillary expansion and facemask therapy followed by fixed appliances. Am J Orthod Dentofacial Orthop,2003 vol. 123, no. 3, pp. 306-320.

14. Ngan P., Wei S. H., Hagg U., Yiu K. Y., Merwin D., and Stickel B. Effect of protraction headgear on class III malocclusion. Quintessence International, 1992;vol. 23, no. 3, pp. 197-207.

15. Ciger S. Effects of 2 types of facemasks on condylar position. Am J Orthod Dentofac Orthop. 2010;137 (6):801-8.

16. De Clerck H, Nguyen T, Paula L,c and Cevidanesd L. Threedimensional assessment of mandibular and glenoid fossa changes after bone-anchored Class III intermaxillary traction. Am J Orthod Dentofacial Orthop 2012; 142:25-31.

17. Lee H, Sung Son1 W, Kwak C, Hee Kang E, Seong- Kim S, Byung Park S and Kim Y. Three-dimensional changes in the temporomandibular joint after maxillary protraction in children with skeletal Class III malocclusion. Journal of Oral Science, 2016; Vol. 58, No. 4, 501-508.

18. Huang $x$, Cen $x$ and Liu J. Effect of protraction facemask on the temporo mandibul ar joint: a systematic review. BMC Oral Health, 2018; Mar 12;18(1):38.

19. Lewis EL, Dolwick MF, Abramowicz S and Reeder SL. Contemporary imaging of the temporomandibular joint. Dent Clin North Am. 2008 ;Oct; 52(4):875-90.

20. Halazonetis DJ. From 2-dimensional cephalograms to 3dimensional computed tomography scans. Am J Orthod Dentofacial Orthop. 2005; 127:627-637.

21. El-Beialy AR, Fayed MS, El-Bialy AM, Mostafa YA. Accuracy and reliability of cone-beam computed tomography measurements: Influence of head orientation. Am J Orthod Dentofacial Orthop. 2011 Aug; 140(2):157-65.

22. Hassel B and Farman Ag. Skeletal maturation evaluation using cervical vertebrae. Am J orthod Dentofac Orthop, 1995;107:58-66.

23. Alhammadi MS, Shafey AS, Fayed MS and Mostafa YA. Temporomandibular joint measurements in normal occlusion: A three-dimensional cone beam computed tomography analysis. J. World Fed. Orthod, 2014;3 , 155-162.

24. Alfeky HY. Three dimensional evaluation of the condylar changes after mandibular protraction for class 2 growing patients. PhD Thesis, Orthodontic Department, Faculty of Oral and Dental Medicine, Cairo University. 2014.

25. Baccetti T, McGill JS, Franchi L and McNamara JA, Tollaro I. Skeletal effects of early treatment of Class III malocclusion with maxillary expansion and face-mask therapy. Am J Orthod Dentofacial Orthop. 1998; Mar;113(3):333-43.

26. Gallagher RW, Miranda F and Buschang PH. Maxillary protraction: treatment and posttreatment effects. Am J Orthod Dentofacial Orthop. 1998; Jun;113(6):612-9.

27. Arman A, Toygar TU and Abuhijleh E. Evaluation of maxillary protraction and fixed appliance therapy in Class III patients. Eur J Orthod. 2006.Aug;(4):383-92. 